\title{
X-ray phase contrast imaging \\ using a grating interferometer
}

Franz Pfeiffer ${ }^{1}$, Timm Weitkamp ${ }^{2}$, and Christian David

${ }^{1}$ Paul Scherrer Institut • 5232 Villigen PSI • Switzerland • ${ }^{2}$ Forschungszentrum Karlsruhe 76021 Karlsruhe • Germany.

$\mathrm{I}^{\mathrm{n}}$

T conventional x-ray imaging, contrast is obtained through the differences in the absorption cross section of the constituents of the object. The technique yields excellent results where highly absorbing structures, e.g., bones, are embedded in a matrix of relatively weakly absorbing material, e.g., the surrounding tissue of the human body. However, in those cases where different forms of tissue with similar absorption crosssections are under investigation (e.g., mammography or angiography), the $\mathrm{x}$-ray absorption contrast is relatively poor. Consequently, differentiating pathologic from non-pathologic tissue from an absorption radiograph obtained with a current hospital-based $\mathrm{x}$-ray system still remains practically impossible for many tissue compositions.

To overcome these limitations, several methods to generate radiographic contrast from the phase shift of $\mathrm{x}$-rays passing through the sample have been investigated in recent years [1-3]. They can be classified into crystal interferometer methods, techniques using an analyzer, and free-space propagation methods. Although some of them yield excellent results for specific problems, none is very widely used. In particular, none of them has so far found medical diagnostics applications, which require a large field of view of many centimeters, the efficient use of broadband radiation as provided by laboratory X-ray generators and a reasonably compact setup.

As an alternative approach we have recently developed a grating based differential phase contrast (DPC) setup which can efficiently be used to retrieve quantitative phase images with polychromatic $\mathrm{x}$-ray sources of low brilliance [4]. Some of the recent results are reviewed in the following.

\section{Principles of Grating Interferometry}

A setup for grating based DPC imaging essentially consists of a phase grating $\mathrm{G} 1$ and an analyzer absorption grating $\mathrm{G}_{2}$ (Fig. 1a). The DPC image formation process is similar to differential interference contrast (DIC) microscopy used with visible light. It essentially relies on the fact that a phase object placed in the x-ray beam path causes slight refraction of the beam transmitted through the object. The fundamental idea of DPC imaging depends on locally detecting these angular deviations (Fig. 1a). The angle, $\alpha$, is directly proportional to the local gradient of the object's phase shift and can be quantified by [5]

$$
\alpha=\frac{\lambda}{2 \pi} \frac{\partial \Phi(x, y)}{\partial x}
$$

where $x$ and $y$ are the Cartesian coordinates perpendicular to the optical axis, $\Phi(x, y)$ represents the phase shift of the wave front, and $\lambda$ the wavelength of the radiation. For hard $x$-rays, with $\lambda<0.1$ $\mathrm{nm}$, the angle is relatively small, typically of the order of a few micro radian.

In our case, determination of the angle is achieved by the arrangement formed by $G_{1}$ and $G_{2}$. Most simply, it can be thought of as a multi-collimator translating the angular deviations into changes of the locally transmitted intensity, which can be detected with a standard imaging detector. For weakly absorbing objects, the detected intensity is a direct measure of the object's local phase gradient $d \Phi(x, y) / d x$. The total phase shift of the object can thus be
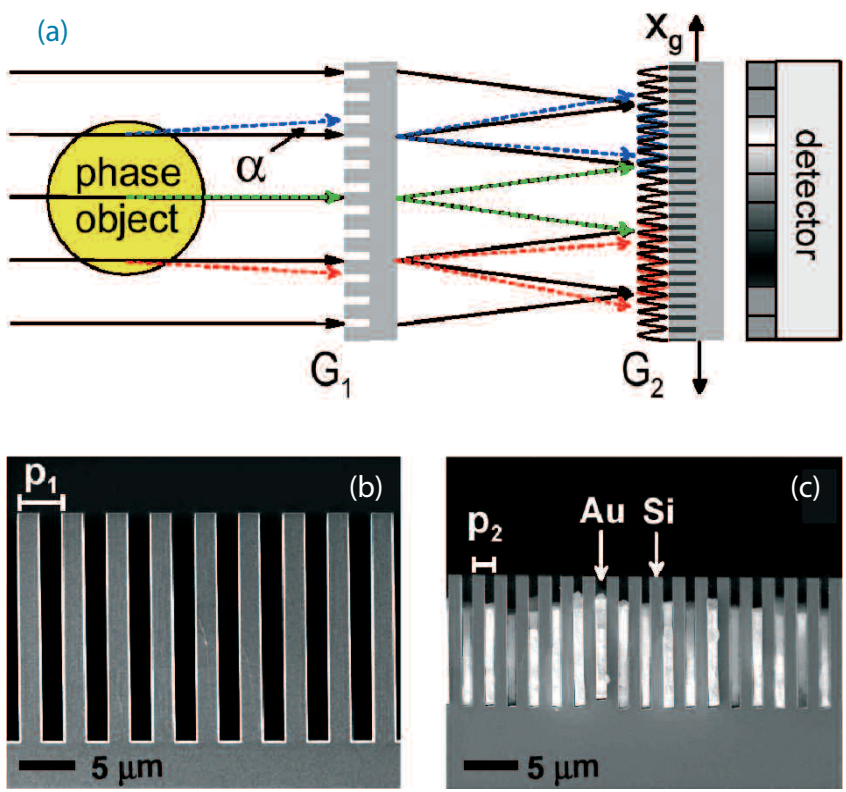

$\Delta$ Fig. 1: (a) Principles of a hard x-ray imaging interferometer. A phase object in the beam path causes a slight refraction for each coherent subset of $x$-rays, which is proportional to the local differential phase gradient of the object. This small angular deviation results in changes of the locally transmitted intensity through the combination of gratings $G_{1}$ and $G_{2}$. A standard x-ray imaging detector is used to record the final images. (b, c) Scanning electron micrograph cross sections of the gratings fabricated by photo lithography, wet chemical etching and electroplating of gold.

V Fig. 2: X-ray images of a rat heart obtained at a highly brilliant x-ray synchrotron source (ESRF, Grenoble).

(a) Conventional X-ray transmission image. (b) Differential phase contrast image.

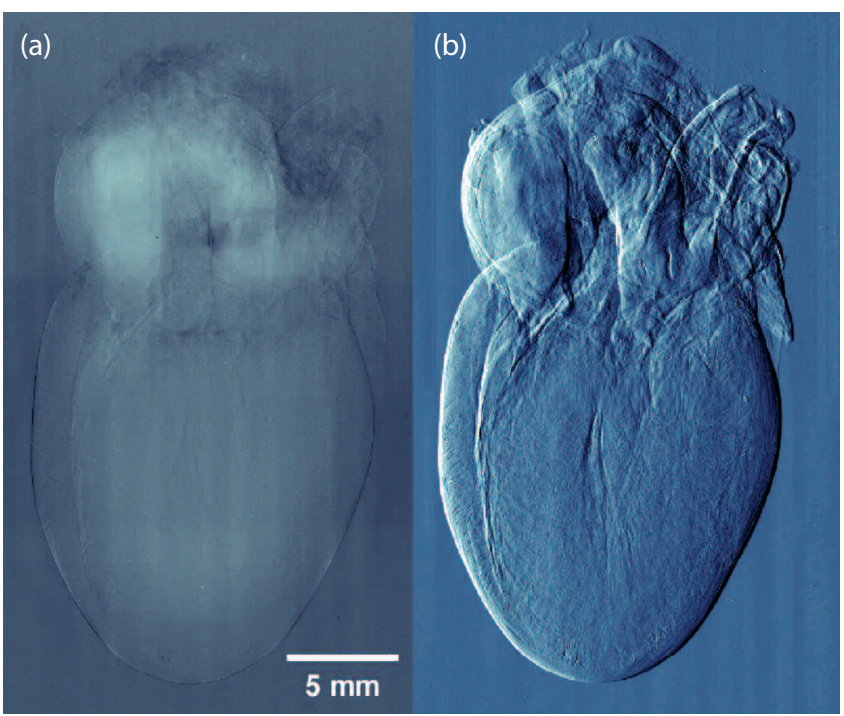


retrieved by a simple one-dimensional integration along $x$. As described in more detail in [6], higher precision of the measurement can be achieved by splitting a single exposure into a set of images taken for different positions of the grating $\mathrm{G}_{2}$.

\section{X-ray Synchrotron Results}

In a first step, the method described was tested using a highly brilliant x-ray synchrotron beam produced by an undulator source at the European Synchrotron Radiation Facility (ESRF, Grenoble, France). As an example, Figure 2 shows the results for an animal organ, a rat heart, which was placed in a container filled with a $4 \%$ aqueous formalin solution. For the experiments monochromatic $\mathrm{x}$-rays of $17.5 \mathrm{keV}$ have been used. In the conventional absorption contrast image (Fig. 2a), only faint details of the fatty tissue and some contrast from the edges of the object are visible. The complete organ with many details on the detailed blood vessel structure can be seen in the differential phase contrast image (Fig. 2b). As is shown in more detail in [5] the DPC images can be quantified and extended to a three-dimensional imaging method by recording projections of the sample for different angles in combination with computer tomographic reconstruction algorithms. In such a way three- dimensional phase images with a resolution of a few micrometers were obtained.

\section{Into the clinics?}

A prerequisite for methods aiming towards clinical $x$-ray applications is that they should work with standard and commercially available $\mathrm{x}$-ray generators, and not only with highly brilliant and partially coherent X-rays from a synchrotron. As we have shown recently [4], in this particular aspect the grating interferometer is superior to already existing phase-sensitive techniques, because it can be adapted for low-brilliance sources. The trick is to use an arrayed source [7], and the easiest way to realize this is by using a third grating $\left(\mathrm{G}_{0}\right)$ just behind the source, as illustrated in Figure 3.

(a)

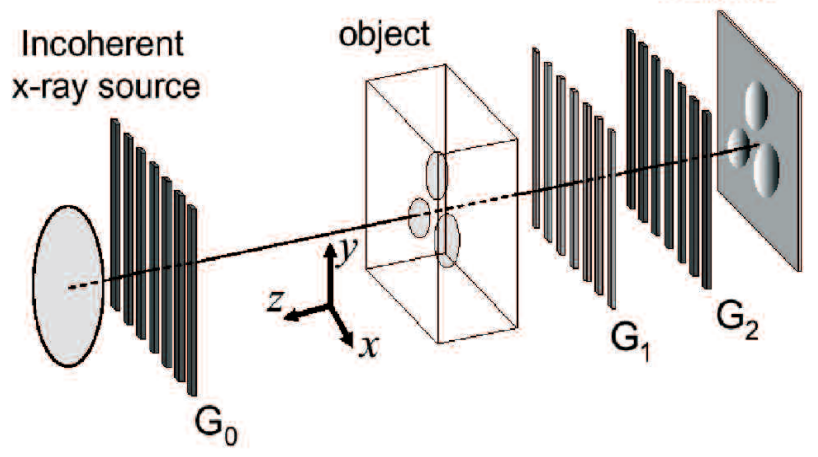

(b)

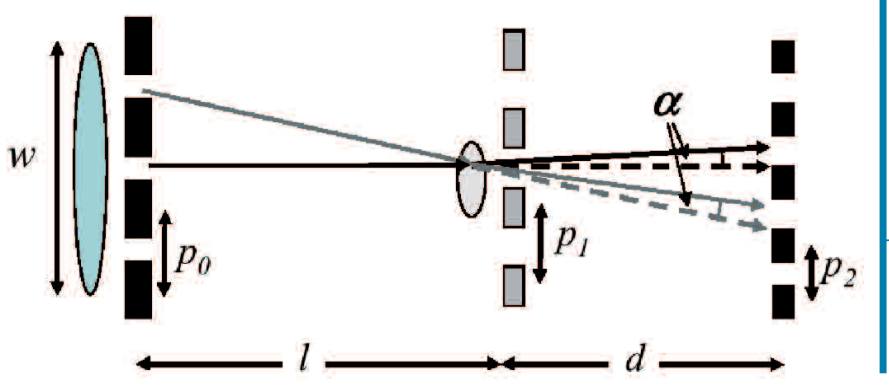

Since the source mask $\mathrm{G}_{0}$ can contain a large number of individual apertures, each creating a sufficiently coherent virtual line source, efficient use can be made of standard x-ray generators with source sizes of more than a square millimetre [4]. To ensure that each line source produced by $\mathrm{G}_{0}$ contributes constructively to the image formation process, the geometry of the setup should satisfy the condition (Fig. 3b)

$$
p_{0}=p_{2} \times \frac{l}{d}
$$

It is important to note that the total source size $w$ only determines the final imaging resolution, which is given by $w d / l$. It does not affect contrast or efficiency, as would be the case for most other phase-sensitive methods.

Figure 4 displays the results of applying our method to a biological object, a small fish (Pterophyllum scalare) using a standard, laboratory based $\mathrm{x}$-ray tube generator. The conventional $\mathrm{x}$-ray transmission image is shown in Fig. 4a, while Fig. 4b contains a greyscale image of the corresponding DPC signal. Since both images were extracted from the same data set, the total exposure time, and thus the dose delivered to the sample was identical in the two cases. The field of view in this case is $50 \times 50 \mathrm{~mm}^{2}$. As expected, the skeleton of the fish and other highly absorbing structures, such as the calcified ear stones (otoliths) are clearly visible in the conventional radiograph (Fig. 4a). However, small differences in the density of the soft tissue, e.g., the different constituents of the eye, are hardly visible in conventional absorption image. In the corresponding DPC image (Fig. 4b), they are clearly visible. We observe that in particular smaller structures with higher spatial frequencies, e.g. the fine structure of the tail fin, are better represented in the DPC image than in the corresponding absorption radiograph.

\section{Conclusion \& Outlook}

These recent results represent a major step forward in radiography with standard $\mathrm{x}$-ray tube sources that could provide all of the information imparted by conventional radiography, with additional information on soft tissue. This may prove to be of clinical importance, particularly in the detection of soft tissue pathologies. This hope is further supported by the fact that DPC methods generally exhibit advantages in the imaging of tumor masses with relatively slow variations of the integrated phase shift if compared to other phase contrast imaging methods, e.g., free space propagation [8].

Due to the fact that the results have been obtained with a standard, relatively low-cost and commercially available $\mathrm{x}$-ray tube generator, we envision a widespread application of our method in areas where phase imaging would be desirable, but is currently unavailable. For example, we believe that this method can readily be implemented without major changes to currently existing medical imaging systems, particularly in the view of the ease of fabricating large-area gratings using standard photolithography, the high resistance of the method against mechanical instabilities, and the possibility to use detectors with large pixels and a large field of view. Since phase contrast imaging does not intrinsically rely on the absorption of $\mathrm{x}$-rays in material, the radiation dose can potentially be reduced by using higher $\mathrm{x}$-ray energies. Furthermore, we envisage applications in the field of

A Fig. 3: (a, b) Setup for $x$-ray tubes. Using a third grating, efficient use can be made of a standard, low-brilliance $x$-ray tube generator with large source sizes. 
non-destructive testing, such as, e.g., the characterization of surface non-uniformities of reflective x-ray optics [9]. Finally, these results open up the way for phase imaging experiments using other forms of radiation, for which only sources of relatively low brilliance currently exist, as for example beams of neutrons [10] or atoms [11].

\section{Acknowledgements:}

We gratefully acknowledge the help of O. Bunk, C. Kottler, J. Bruder, E. Ziegler, and P. Cloetens. This work was partly supported by the KTI under contract 7796.2 DCPP-NM.

\section{About the authors:}

Franz Pfeiffer studied Physics at the University in Munich. During his $\mathrm{PhD}$ he worked on X-Ray and neutron waveguides at the Institut Laue Langevin and the European Synchrotron Radiation Facility (ESRF) in Grenoble. Since then he is leading a research group on coherent $\mathrm{x}$-ray scattering at the Paul Scherrer Institut (PSI).

Timm Weitkamp studied Physics at the University of Hamburg. He received his $\mathrm{PhD}$ in 2002 for a thesis on high-resolution microtomography and hard X-ray microscopy at the ESRF. After a PostDoc stay at the PSI, he is now a beamline scientist at the ANKA light source operated by Forschungszentrum Karlsruhe

Christian David studied Physics at the University of Göttingen. During his $\mathrm{PhD}$, he worked on $\mathrm{x}$-ray microscopy and nanolithography techniques. After a PostDoc stay at the Institute for Applied Physical Chemistry, University of Heidelberg, he joined the PSI where he presently leads the X-ray Optics and Applications Group.

\section{References}

[1] R. Fitzgerald, Physics Today 53, 23 (2000).

[2] A. Momose, Optics Express 11, 2303 (2003).

[3] A. Momose, Jap. Jour. Appl. Phys. 44, 6355 (2005).

[4] F. Pfeiffer, T. Weitkamp, O. Bunk, and C. David, Nature Physics 2, 258 (2006).

[5] T. Weitkamp, A. Diaz, C. David, F. Pfeiffer, M. Stampanoni, P. Cloetens, and E. Ziegler, Optics Express 13, 6296 (2005).

[6] T. Weitkamp, A. Diaz, B. Nöhammer, F. Pfeiffer, T. Rohbeck, P. Cloetens, and C. David, Proc. SPIE 5535, 137 (2004).

[7] C. David, F. Pfeiffer, and T. Weitkamp, European patent application 05012121.9 / EP05012121 (2005).

[8] E. Pagot, S. Fiedler, P. Cloetens, A. Bravin, P. Coan, K. Fezzaa, J. Baruchel, and J. Härtwig, Phys. Med. Biol. 50, 709 (2005).

[9] T. Weitkamp, B. Nöhammer, A. Diaz, C. David, and E. Ziegler, Appl. Phys. Lett. 86, 054101 (2005).

[10] F. Pfeiffer, C. Grünzweig, O. Bunk, G. Frei, E. Lehmann, and C. David, Phys. Rev. Lett. 96, 215505 (2006).

[11] D.W. Keith, C.R. Ekstrom, Q.A. Turchette, and D.E. Pritchard, Phys. Rev. Lett. 66, 2693 (1991).

V Fig. 4: X-ray images of a small fish retrieved from image data recorded with a standard x-ray tube operated at $40 \mathrm{kV} / 25 \mathrm{~mA}$

(a) Conventional X-ray transmission image. (b) Differential phase contrast image. The total exposure time was 200 seconds. 